\title{
FREE LATTICES WITH INFINITE OPERATIONS
}

\author{
BY \\ PETER CRAWLEY AND RICHARD A. DEAN
}

1. Introduction. A lattice $L$ is said to be $\mathfrak{n}$-complete for a given cardinal $\mathfrak{n}$, if every subset of $L$ of cardinality at most $\mathfrak{n}$ has a least upper bound and a greatest lower bound in $L$. Every lattice is necessarily $\mathfrak{n}$-complete for all finite $\mathfrak{n}$, but need not be $\mathfrak{n}$-complete for any infinite cardinal $\mathfrak{n}$. Free lattices offer examples of this. A more general concept of completeness is that of weak $\mathfrak{n}$-completeness. A lattice $L$ is called weakly $\mathfrak{n}$-complete for a given cardinal $\mathfrak{n}$, if every subset of $L$ of cardinality less than $\mathfrak{n}$ has a least upper bound and a greatest lower bound in L. Again, every lattice, in particular a free lattice, is weakly $\boldsymbol{\aleph}_{0}$-complete. Evidently a lattice is $\boldsymbol{\aleph}_{\alpha}$-complete if and only if it is weakly $\boldsymbol{\aleph}_{\alpha+1}$-complete.

The purpose of this paper is to study generalizations of the ordinary free lattices which have this property of weak $\boldsymbol{\aleph}_{\alpha}$-completeness. In order to define these "generalized free lattices" the following ideas are needed.

Let $L$ be a weakly $\boldsymbol{\aleph}_{\alpha}$-complete lattice. $A$ sublattice $M$ of $L$ is an $\alpha$-sublattice if meets and joins in $L$ of subsets of $M$ of cardinality less than $\aleph_{\alpha}$ are also contained in $M$. If $S$ is a subset of $L$, then the sublattice of $L \alpha$-generated by $S$ is the smallest $\alpha$-sublattice of $L$ containing $S$. In particular, $L$ is said to be $\alpha$-generated by $S$ if the only $\alpha$-sublattice of $L$ containing $S$ is just $L$ itself.

$A$ lattice $F$ is freely $\alpha$-generated by a partially ordered set $P$, if $F$ contains $P$ as a subpartially ordered set and is $\alpha$-generated by it, and if for any lattice $L \alpha$-generated by a subset $Q$, every order preserving mapping of $P$ onto $Q$ extends to a homomorphism (preserving meets and joins of less than $\boldsymbol{\aleph}_{\alpha}$ elements) of $F$ onto $L$. It is easily seen that $F$ is unique up to isomorphism.

The lattice freely $\alpha$-generated by $P$ is constructed in the second section of the paper. The techniques used here are extensions of those employed by Whitman $[6 ; 7]$ in describing the ordinary free lattice with an unordered set of generators. The analogue of the result of Whitman that the free lattice with three generators contains as a sublattice the free lattice with a countable number of generators is proved in section three, namely, it is shown that the lattice freely $\alpha$-generated by three unordered elements contains the lattice freely $\alpha$-generated by $\boldsymbol{\aleph}_{\alpha}$ unordered elements as an $\alpha$-sublattice. This result is used to show that the lattice freely $\alpha$-generated by a partially ordered set of cardinality at most $\boldsymbol{\aleph}_{\alpha}$ is isomorphic to an $\alpha$-sublattice of the lattice freely $\alpha$ generated by three unordered elements.

Recently Bjarni Jónsson [4] has given a nonconstructive proof that under the assumption of the Generalized Continuum Hypothesis, $\boldsymbol{\aleph}_{\alpha}$-universal lat-

Received by the editors January 25, 1958. 
tices $\left.{ }^{1}\right)$ exist for all $\alpha>0$, and $\boldsymbol{\aleph}_{\alpha}$-universal partially ordered sets exist for all ordinals $\alpha$. In the fourth section, it is first shown that any lattice of cardinality at most $\boldsymbol{\aleph}_{\alpha}$ is a sublattice of a lattice $\alpha$-generated by three elements, and the method of proof of this theorem is used to show that $\boldsymbol{\aleph}_{0}$-universal lattices do not exist. It is further proved that the free lattice with three generators is an $\mathbf{\aleph}_{0 \text { - }}$ universal partially ordered set. Under the Generalized Continuum Hypothesis, the lattice freely $\alpha$-generated by three unordered elements is an $\boldsymbol{\aleph}_{\alpha}$-universal partially ordered set if $\boldsymbol{\aleph}_{\alpha}$ is regular, and contains a sublattice which is an $\boldsymbol{\aleph}_{\alpha-}$ universal partially ordered set if $\boldsymbol{\aleph}_{\alpha}$ is singular.

2. The lattice freely $\alpha$-generated by $P$. Throughout the paper the following notation will be used. Lattice join, meet, inclusion and proper inclusion are denoted respectively by $\cup, \cap$, $\leqq,<$, with the symbols $+\left(\sum\right), \cdot(\Pi)$, $\subseteq, \subset$, reserved for the corresponding set operations. The cardinality of a set $\mathfrak{T}$ is denoted by $|\mathfrak{T}|$. For a given cardinal $\mathfrak{n}, \omega(\mathfrak{n})$ denotes the least ordinal of cardinality $\mathfrak{n} ; \omega\left(\boldsymbol{\aleph}_{\alpha}\right)$ is briefly written $\omega_{\alpha}$.

We begin with an alternative recursive definition of the sublattice $\alpha$ generated by a subset $S$ of a weakly $\boldsymbol{\aleph}_{\alpha}$-complete lattice $L$. Let $\beta=\omega_{\alpha}$ if $\omega_{\alpha}$ is regular or $\beta=\omega_{\alpha+1}$ if $\omega_{\alpha}$ is singular $\left({ }^{2}\right)$. With $M_{0}=S$, define $M_{\xi}$ for all $\xi \leqq \beta$ by

$M_{\xi}=\left\{\cup a, \cap a \in L\left|a \subseteq M_{\xi-1},\right| a \mid<\boldsymbol{N}_{\alpha}\right\}, \quad$ if $\xi$ is not a limit ordinal, $M_{\xi}=\sum_{n<\xi} M_{n}, \quad$ if $\xi$ is a limit ordinal.

Then $M_{\beta}$ is the sublattice of $L \alpha$-generated by $S$.

Now let $P$ be a partially ordered set, and $\alpha$ an ordinal. $\alpha$-words over $P$ and their ranks are defined inductively as follows.

Definition 1. (1) For each $p \in P, p$ is an $\alpha$-word over $P$, and its rank $r(p)$ is zero.

(2) If $Q$ is a nonempty set of $\alpha$-words over $P$ such that $|a|\left\langle\boldsymbol{\aleph}_{\alpha}\right.$, then the symbols $\cup Q$ and $\cap Q$ are $\alpha$-words over $P$, and

$$
r(\cup Q)=r(\cap Q)=\max \left\{\omega(|Q|), \sup _{A \in Q}[r(A)+1]\right\} .
$$

The set of all $\alpha$-words over $P$ is denoted by $F_{\alpha}(P)$.

Definition 2. If $A, B \in F_{\alpha}(P)$, then $A \geqq B$ if one of the conditions below $\operatorname{holds}\left({ }^{3}\right)$.

(1) $A, B \in P$ and $A \geqq B$ in $P$.

(2) (i) $A \equiv \cup Q$ and $A^{\prime} \geqq B$ for some $A^{\prime} \in Q$,

(ii) $A \equiv \cap \propto$ and $A^{\prime} \geqq B$ for all $A^{\prime} \in Q$,

(1) For the definitions of these terms, see $\$ 4$.

(2) $\boldsymbol{\aleph}_{\alpha}$ and $\omega_{\alpha}$ are said to be singular, if $\boldsymbol{\aleph}_{\alpha}$ is the sum of fewer than $\boldsymbol{\aleph}_{\alpha}$ cardinals, each of which is less than $\boldsymbol{\aleph}_{\alpha}$. Otherwise, $\boldsymbol{\aleph}_{\alpha}$ and $\omega_{\alpha}$ are said to be regular.

$\left.{ }^{3}\right)(\equiv)$ denotes logical identity. 
(iii) $B \equiv \bigcup \Theta$ and $A \geqq B^{\prime}$ for all $B^{\prime} \in \Theta$,

(iv) $B \equiv \bigcap \leftrightarrow$ and $A \geqq B^{\prime}$ for some $B^{\prime} \in B$.

Definition 3. If $A, B \in F_{\alpha}(P)$, then $A=B$ if $A \geqq B$ and $B \geqq A$.

Lemma 1. Let $A \equiv \cup \propto, B \equiv \cap \circledast$, and $C$ be $\alpha$-words over $P$. Then:

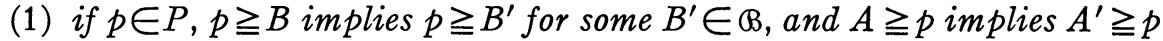
for some $A^{\prime} \in Q$;

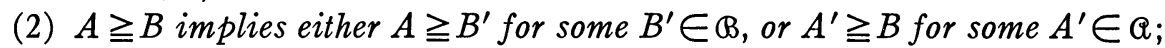

(3) $C \geqq A$ implies $C \geqq A^{\prime}$ for all $A^{\prime} \in Q ; B \geqq C$ implies $B^{\prime} \geqq C$ for all $B^{\prime} \in ß$.

Proof. (1) and (2) are immediate from Definition 2. The first half of (3) is proved by induction on $r(C)$, the other half following by duality. If $C \in P$, then $C \geqq A$ implies $C \geqq A^{\prime}$ for all $A^{\prime} \in Q$, since (iii) is the only applicable rule of Definition 2. Assuming the result for $r\left(C^{\prime}\right)<\eta$, let $r(C)=\eta$ and $C \geqq A$. If $C \equiv \mathrm{U} \mathcal{C}$, either (i) or (iii) of Definition 2 holds. (iii) gives the result. (i) states that $C^{\prime} \geqq A$ for some $C^{\prime} \in \mathrm{e}$, and since $r\left(C^{\prime}\right)<r(C)$, the induction implies that $C^{\prime} \geqq A^{\prime}$, all $A^{\prime} \in Q$. Thus $C \geqq A^{\prime}$ for all $A^{\prime} \in Q$ follows from (i). A similar argument holds for $C \equiv$ กe.

THEOREM 1. $F_{\alpha}(P)$ is a lattice under the relation ( $\geqq$ ) of Definition 2. Moreover, if $a$ is a nonempty subset of $F_{\alpha}(P)$, with $|Q|\left\langle\boldsymbol{N}_{\alpha}\right.$, then the $\alpha$-words $\cup a$ and $\cap Q$ are respectively the least upper and greatest lower bounds of $a$ in $F_{\alpha}(P)$.

Proof. The relation $(\geqq)$ is first shown to be reflexive and transitive.

(1.1) For all $A \in F_{\alpha}(P), A \geqq A$.

If $A \in P$, the result follows from the reflexive law in $P$. Inducting on $r(A)$, suppose (1) holds whenever $r\left(A^{\prime}\right)<\eta$, and let $r(A)=\eta$. If $A \equiv \mathrm{U} Q$, then $A^{\prime} \geqq A^{\prime}$ for all $A^{\prime} \in Q$, so that (1.1) follows from (i) and (iii) of Definition 2 . A dual argument holds if $A \equiv \cap$ Q.

(1.2) $A \geqq B$ and $B \geqq C$ imply $A \geqq C$.

The proof of (1.2) is by an induction on the ordered triples $\langle r(A), r(B)$, $r(C)\rangle$, ordered lexicographically. When $\langle r(A), r(B), r(C)\rangle=\langle 0,0,0\rangle,(1.2)$ follows from the transitive law in $P$. Suppose that (1.2) holds for any triple less than $\left\langle\eta, \eta^{\prime}, \eta^{\prime \prime}\right\rangle$, and that $\langle r(A), r(B), r(C)\rangle=\left\langle\eta, \eta^{\prime}, \eta^{\prime \prime}\right\rangle$ with $A \geqq B, B \geqq C$. There are ten cases to consider: (i) $A, B \in P, C \equiv \cup \mathfrak{e}$; (ii) $A, B \in P, C \equiv \cap \mathfrak{e}$; (iii) $B \in P, A \equiv \bigcup_{Q}$; (iv) $B \in P, A \equiv \cap Q$; (v) $B \equiv \bigcup_{B}, C \in P$; (vi) $B \equiv \bigcup_{B}$, $C \equiv \mathrm{Ue}$; (vii) $B \equiv \mathrm{U} \leftrightarrow, C \equiv \cap \mathfrak{e}$; and the duals of (v), (vi), and (vii). We shall give the proofs of (i) and (vii); the proofs of the remaining cases are very similar to these.

(i) $A, B \in P, C \equiv$ Ue. From $B \geqq C$, it follows that $B \geqq C^{\prime}$ for all $C^{\prime} \in \mathcal{e}$. Now $\left\langle r(A), r(B), r\left(C^{\prime}\right)\right\rangle\left\langle\left\langle\eta, \eta^{\prime}, \eta^{\prime \prime}\right\rangle\right.$, so that $A \geqq C^{\prime}$ for all $C^{\prime} \in \mathfrak{e}$, by the induction hypothesis. Hence $A \geqq C$.

(vii) $B \equiv \bigcup_{B}, C \equiv$ กe. $B \geqq C$ implies either $B \geqq C^{\prime}$ for some $C^{\prime} \in \mathcal{C}$, or $B^{\prime \prime} \geqq C$ for some $B^{\prime \prime} \in$ B. If the first alternative holds, then $A \geqq C^{\prime}$, and hence

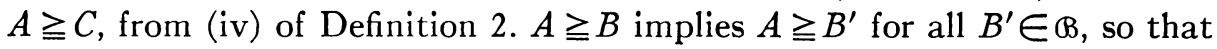


in particular, $A \geqq B^{\prime \prime}$. If the second alternative holds, then $A \geqq C$ follows from the induction hypothesis.

From (1.1) and (1.2) it follows that $(=)$ of Definition 3 is an equivalence relation. Hence the relation ( $\geqq$ ) partially orders the set of all $\alpha$-words over $P$.

Now consider a nonempty set $Q$ with $|a|\left\langle\boldsymbol{\aleph}_{\alpha}\right.$. Then $U Q$ and $\cap Q$ are $\alpha$-words over $P$, and $\cup Q \geqq A^{\prime} \geqq \cap Q$ for every $A^{\prime} \in Q$. Moreover, if $B \geqq A^{\prime} \geqq C$ for all $A^{\prime} \in Q$ then $B \geqq \cup Q$ and $\cap Q \geqq C$, from (iii) and (ii) of Definition 2 . Thus the least upper and greatest lower bounds of $Q$ exist and are indeed $U Q$ and $\cap Q$ respectively.

For convenience, the $\alpha$-words $\cup\left\{A_{1}, A_{2}\right\}$ and $\cap\left\{A_{1}, A_{2}\right\}$ will also be written $A_{1} \cup A_{2}$ and $A_{1} \cap A_{2}$ respectively. It must be emphasized that $A_{1} \cup A_{2}$, $A_{2} \cup A_{1}$, and $\cup\left\{A_{1}, A_{2}\right\}$ are to be considered as logically identical $\alpha$-words.

In view of Definition 2, $P$ is certainly a subpartially ordered set of $F_{\alpha}(P)$. However, least upper and greatest lower bounds existing in $P$ need not be preserved in $F_{\alpha}(P)$. If $p \in P$ and $a \subseteq P$ is such that $|a|\left\langle\boldsymbol{\aleph}_{\alpha}\right.$ and $p=1$.u.b. $a$ (or g.l.b. $Q$ ) in $P$, then $p=\cup Q(\cap Q)$ if and only if $p=p^{\prime}$ for some $p^{\prime} \in Q$.

THEOREM 2. $F_{\alpha}(P)$ is freely $\alpha$-generated by $P$.

Proof. That $P \alpha$-generates $F_{\alpha}(P)$ is clear from the construction of $F_{\alpha}(P)$. To show the free property, consider a lattice $L \alpha$-generated by a subpartially ordered set $Q$, and an order-preserving mapping $\phi$ of $P$ onto $Q$. Extend this mapping to a mapping $\phi^{*}$ of $F_{\alpha}(P)$ into $L$ inductively as follows:

$$
\begin{gathered}
\phi^{*}(A)=\phi(A) \quad \text { if } A \in \\
\phi^{*}(\cup a)=\bigcup\left\{\phi^{*}\left(A^{\prime}\right) \mid A^{\prime} \in a\right\}, \quad \phi^{*}(\cap a)=\bigcap\left\{\phi^{*}\left(A^{\prime}\right) \mid A^{\prime} \in a\right\} .
\end{gathered}
$$

Since $\phi$ maps $P$ onto $Q$, and since $L$ is $\alpha$-generated by $Q, \phi^{*}$ is a mapping of $F_{\alpha}(P)$ onto $L$. To show that $\phi^{*}$ is a homomorphism, we need only show that it preserves the equality in $F_{\alpha}(P)$, or what is equivalent, that $A \geqq B$ implies $\phi^{*}(A) \geqq \phi^{*}(B)$. This is done by an induction on the pairs $\langle r(A), r(B)\rangle$, ordered lexicographically.

If $A, B \in P$, then $A \geqq B$ implies $\phi^{*}(A)=\phi(A) \geqq \phi(B)=\phi^{*}(B)$, since $\phi$ preserves the ordering of $P$. Continuing the induction, let $A \equiv \cap \propto . A \geqq B$ implies $A^{\prime} \geqq B$ and hence $\phi^{*}\left(A^{\prime}\right) \geqq \phi^{*}(B)$, for all $A^{\prime} \in Q$; whence $\phi^{*}(A)=\phi^{*}\left(\cap_{Q}\right)$ $\geqq \phi^{*}(B)$. A dual proof holds if $B \equiv \cup ß$. For $A \in P, B \equiv \cap \AA, A \geqq B$ implies that $A \geqq B^{\prime \prime}$ for some $B^{\prime \prime} \in B$, so that $\phi^{*}(A) \geqq \phi^{*}\left(B^{\prime \prime}\right) \geqq \bigcap\left\{\phi^{*}\left(B^{\prime}\right) \mid B^{\prime} \in B\right\} \equiv \phi^{*}(B)$. Dually, if $A \equiv \cup \propto$ and $B \in P$. Finally if $A \equiv \cup a$ and $B \equiv \cap \circledast, A \geqq B$ implies either $A \geqq B^{\prime}$ for some $B^{\prime} \in B$ or $A^{\prime} \geqq B$ for some $A^{\prime} \in Q$. But then either $\phi^{*}(A) \geqq \phi^{*}\left(B^{\prime}\right) \geqq \phi^{*}(B)$ or $\phi^{*}(A) \geqq \phi^{*}\left(A^{\prime}\right) \geqq \phi^{*}(B)$. Hence $\phi^{*}$ preserves the ordering of $F_{\alpha}(P)$, and the proof is complete.

At this point it is well to note that $F_{0}(P)$ is just the completely free lattice generated by $P$, studied in [2]. Thus when $P$ is an unordered set of cardinaliiy $\mathfrak{n}, F_{0}(P)$ is the familiar free lattice with $\mathfrak{n}$ generators, discussed by Whitman $[6 ; 7]$. 
Theorem 3. If $\boldsymbol{\aleph}_{\alpha}$ is a singular cardinal, then $F_{\alpha}(P)$ and $F_{\alpha+1}(P)$ are isomorphic lattices.

Proof. $F_{\alpha}(P)$ is an $\alpha$-sublattice of $F_{\alpha+1}(P)$, so it suffices to show that for each $A \in F_{\alpha+1}(P)$ there exists $B \in F_{\alpha}(P)$, such that $A=B$. When $r(A)=0$,

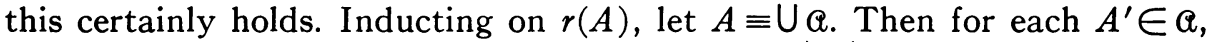
there exists $B_{A^{\prime}} \in F_{\alpha}(P)$ such that $A^{\prime}=B_{A^{\prime}}$. Now $|Q| \leqq \aleph_{\alpha}$, and since $\aleph_{\alpha}$ is singular, $Q$ can be partitioned up into a set $\subseteq$ of subsets $\delta \subseteq Q$ in such a way that $|\mathfrak{S}|<\aleph_{\alpha}$ and $|\mathfrak{s}|<\aleph_{\alpha}$ for each $s \in \mathfrak{S}$. Thus $B_{\mathcal{S}} \equiv \cup\left\{B_{A^{\prime}} \mid A^{\prime} \in \mathcal{S}\right\} \in F_{\alpha}(P)$ for each $\mathcal{S} \in \mathfrak{S}$, and hence $B \equiv \bigcup\left\{B_{\mathcal{S}} \mid \delta \in \mathfrak{S}\right\} \in F_{\alpha}(P)$. Moreover, $B=A$. A dual proof holds if $A \equiv \cap \propto$.

\section{Embeddings in freely $\alpha$-generated lattices.}

THEOREm 4. Let $Q$ be a partially ordered set and $\boldsymbol{\aleph}_{\alpha}$ a regular cardinal. If a subpartially ordered set $\mathcal{S}$ of $F_{\alpha}(Q)$ is isomorphic to a partially ordered set $P$, then the sublattice $L_{\mathcal{S}} \alpha$-generated by $S$ in $F_{\alpha}(Q)$ is isomorphic to $F_{\alpha}(P)$ if and only if the following condition and its dual are satisfied:

For each $A \in \mathcal{S}$ and each subset $a \subseteq S$ with $|\propto|<\aleph_{\alpha}, \cup \propto \geqq A$ implies $A^{\prime} \geqq A$ for some $A^{\prime} \in$ Q.

Proof. It is clear from Lemma 1 that the above conditions on $\mathcal{S}$ are necessary for $L_{\mathcal{S}}$ to be isomorphic to $F_{\alpha}(P)$. To show their sufficiency, consider an isomorphic mapping $\phi$ of $P$ onto $\delta$. Since $L_{\mathcal{S}}$ is $\alpha$-generated by $\mathcal{S}, \phi$ can be extended to a homomorphism $\phi^{*}$ of $F_{\alpha}(P)$ onto $L_{\mathcal{S}}$. We shall show that $\phi^{*}$ is one-one and hence an isomorphism. A second lemma is needed.

Lemma 2. Let $\aleph_{\alpha}$ be a regular cardinal. If $p \in P$, and $A \in F_{\alpha}(P)$ is such that $A \geqq p$, then there exists a subset $\mathcal{C} \subset P$ with $|\mathfrak{C}|<\aleph_{\alpha}$ such that $\cup \mathfrak{C} \geqq A$, but Ue

When $A \in P, \mathfrak{e}=\{A\}$ fulfills the conditions of the lemma. Now suppose that Lemma 2 holds for all $\alpha$-words of rank less than $r(A)$. If $A \equiv \mathrm{U} Q$, then $A \notin p$ implies $A^{\prime} \geq p$ for all $A^{\prime} \in Q$. Thus $\mathcal{C}_{A^{\prime}}$ exists for each $A^{\prime} \in Q$, satisfying the conditions of the lemma for $A^{\prime}$ and $p$; take $\mathcal{C}=\sum_{A^{\prime} \in Q} \mathfrak{C}_{A^{\prime}}$. Since $\boldsymbol{\aleph}_{\alpha}$ is regular, $|\mathfrak{e}|<\aleph_{\alpha}$. Furthermore $U \mathcal{C} \geqq A$, but $U \mathfrak{C} \geqq p$. A similar proof holds for the case $A \equiv \cap$ Q.

Now to show that $\phi^{*}$ is one-one, we need only show that $\phi^{*}(A) \geqq \phi^{*}(B)$ implies $A \geqq B$, for all $A, B \in F_{\alpha}(P)$. When $A, B \in P$, this clearly holds. Suppose that this implication holds for any pair less than $\langle r(A), r(B)\rangle$ and that $\phi^{*}(A) \geqq \phi^{*}(B)$. If $B \in P$ and $A \geq B$, then by Lemma 2, $\mathcal{C} \subset P$ exists with $|\mathfrak{e}|<\mathbb{N}_{\alpha}$ such that Ue $\geqq A$ but Ue $B$. Thus $\phi^{*}(\mathrm{Ue})=\bigcup\left\{\phi^{*}(p) \mid p \in \mathfrak{e}\right\}$ $\geqq \phi^{*}(A) \geqq \phi^{*}(B)$. $\left\{\phi^{*}(p) \mid p \in \mathcal{C}\right\} \subset \mathcal{S}$ and $\phi^{*}(B) \in \mathcal{S}$, so that $\phi^{*}(p) \geqq \phi^{*}(B)$ for some $p \in \mathcal{C}$. But $\phi^{*}$ maps $P$ isomorphically onto $\mathcal{S}$, and hence $p \geqq B$. This implies that $\cup \mathbb{C} \geqq B$, a contradiction. The case $A \in P$ follows by a dual argument, and the proofs of the remaining cases are the same as those for the correspond- 
ing cases in Theorem 2 . Hence that $\phi^{*}(A) \geqq \phi^{*}(B)$ implies $A \geqq B$ is true by induction, and $L_{\mathcal{S}}$ and $F_{\alpha}(P)$ are isomorphic lattices.

In the two theorems to follow, lattices freely $\alpha$-generated by unordered sets are important. For each cardinal $\mathfrak{n}$, the lattice freely $\alpha$-generated by $\mathfrak{n} u n$ ordered elements will be denoted by $F_{\alpha}(\mathfrak{n})$. Whitman [7] has shown that $F_{0}(3)$ contains $F_{0}\left(\boldsymbol{\aleph}_{0}\right)$ as a sublattice; Theorem 5 extends this result to arbitrary $\alpha$.

Theorem 5. For each ordinal $\alpha$, and each cardinal $\mathfrak{n} \leqq \boldsymbol{\aleph}_{\alpha}, F_{\alpha}(\mathfrak{n})$ is an $\alpha$-sublattice of $F_{\alpha}(3)$.

Proof. The proof of Theorem 5 is an extension of the proof of Whitman's theorem given by Dilworth [3]. Since $\boldsymbol{\aleph}_{\alpha+1}$ is regular for all $\alpha$, by Theorem 3 it is sufficient to consider only the case when $\boldsymbol{\aleph}_{\alpha}$ is regular.

Let $F_{\alpha}(3)$ be $\alpha$-generated by the three unordered elements, $a, b$, and $c$. For each $A \in F_{\alpha}(3)$, define the $\alpha$-words $f(A)$ and $h(A)$ by

$$
\begin{aligned}
& f(A) \equiv a \cup(b \cap(c \cup(a \cap(b \cup(c \cap A))))), \\
& h(A) \equiv a \cap(b \cup(c \cap(a \cup(b \cap(c \cup A))))) .
\end{aligned}
$$

Setting $x_{0} \equiv y_{0} \equiv a$, and proceeding inductively, define for each ordinal $\xi<\omega_{\alpha}$,

$$
\begin{array}{lll}
x_{\xi} \equiv f\left(x_{\xi-1}\right), & y_{\xi} \equiv h\left(y_{\xi-1}\right), & \text { if } \xi \text { is not a limit ordinal, } \\
x_{\xi} \equiv \cup\left\{x_{\eta} \mid \eta<\xi\right\}, & y_{\xi} \equiv \cap\left\{y_{\eta} \mid \eta<\xi\right\}, & \text { if } \xi \text { is a limit ordinal. }
\end{array}
$$

It is clear that $x_{\xi}$ and $y_{\xi}, \xi<\omega_{\alpha}$, are indeed $\alpha$-words over $\{a, b, c\}$.

(5.1) For all $\xi, 0<\xi<\omega_{\alpha}: x_{\xi} \geq, c ; a, b, c \geq x_{\xi} ; b, c \geq y_{\xi} ;$ and $y_{\xi} \geq a, b, c$.

This holds, since for all $\xi$ with $0<\xi<\omega_{\alpha}$, we have $a \cup(b \cap(c \cup a)) \geqq x_{\xi}$ $\geqq a \cup(b \cap c)$ and $a \cap(b \cup c) \geqq y_{\xi} \geqq a \cap(b \cup(c \cap a))$.

(5.2) If $A, B \in F_{\alpha}(3)$, then $a \cup c \geqq A, B \geqq c$, and $a \cup(b \cap A) \geqq a \cup(b \cap B)$ imply $b \cap A \geqq b \cap B$. And dually.

Let $a \cup(b \cap A) \geqq a \cup(b \cap B) \geqq b \cap B$. Then $a \cup(b \cap A) \geqq b$ implies $a \cup c$ $\geqq a \cup A \geqq b ; a \cup(b \cap A) \geqq B$ implies $a \cup b \geqq c$; and $a \geqq b \cap B$ implies $a \geqq b \cap c$. Since all these are impossible, the only remaining possibility is $b \cap A \geqq b \cap B$. Notice that (5.2) also holds for cyclic permutations of $a, b$, and $c$.

(5.3) If $\eta<\xi<\omega_{\alpha}$, then $x_{\eta}<x_{\xi}$ and $y_{\xi}<y_{\eta}$.

From (5.1) it is clear that $x_{\xi}>x_{0}, 0<\xi<\omega_{\alpha}$. Suppose that $\eta<\mu$ implies $x_{\eta} \leqq x_{\mu}$ whenever $\mu<\xi<\omega_{\alpha}$. If $\xi$ is a limit ordinal, then $x_{\xi} \equiv \bigcup\left\{x_{\eta} \mid \eta<\xi\right\} \geqq x_{\eta}$ for all $\eta<\xi$. When $\xi$ is not a limit ordinal and $\xi-1$ is not a limit ordinal, $x_{\xi-1} \geqq x_{\xi-2}$ implies $x_{\xi} \equiv f\left(x_{\xi-1}\right) \geqq f\left(x_{\xi-2}\right) \equiv x_{\xi-1} \geqq x_{\eta}$ all $\eta<\xi$, since $f$ preserves the ordering. If $\xi-1$ is a limit ordinal, then $x_{\xi} \equiv f\left(x_{\xi-1}\right) \geqq f\left(x_{\eta}\right) \equiv x_{\eta+1}$ for all $\eta<\xi-1$, and hence $x_{\xi} \geqq U\left\{x_{\eta} \mid \eta<\xi-1\right\} \equiv x_{\xi-1} \geqq x_{\eta}$ all $\eta<\xi$. Thus by induction, $x_{\eta} \leqq x_{\xi}$ whenever $\eta<\xi<\omega_{\alpha}$. To show that this inclusion is proper, assume the contrary. Then there is a least ordinal $\xi$ such that $x_{\eta} \geqq x_{\xi}$ for some $\eta<\xi$. $\xi$ cannot be a limit ordinal, since otherwise $x_{\xi} \equiv \bigcup\left\{x_{\mu} \mid \mu<\xi\right\} \leqq x_{\eta}$ implies 
$x_{\eta} \geqq x_{\eta+1}$, contrary to the minimality of $\xi$. Suppose that $\eta$ is not a limit ordinal. Then $x_{\eta} \equiv f\left(x_{\eta-1}\right)$, and successive applications of (5.2) give $x_{\eta-1} \geqq c \cap x_{\eta-1}$ $\geqq c \cap x_{\xi-1}$. If $\eta-1$ is not a limit ordinal, $x_{\eta-1} \geq c$ and $a, b \geq c \cap x_{\xi-1}$ imply that $x_{\eta-1} \geqq x_{\xi-1}$, a contradiction. If $\eta-1$ is a limit ordinal, then $x_{\eta-1} \geq c, x_{\xi-1}$, and hence $x_{\eta_{1}} \geqq c \cap x_{\xi-1}$ for some $\eta_{1}<\eta-1$. Continuing in this way if $\eta_{1}$ is a limit ordinal we get a descending chain of ordinals which must terminate with some ordinal $\eta_{k}$, not a limit ordinal. But then, as above, $x_{\eta_{k}} \geqq c \bigcap x_{\xi-1}$ implies $x_{\eta_{k}} \geqq x_{\xi-1}$, a contradiction. Finally if $\eta$ is a limit ordinal, since $x_{\eta} \geq b, c$, we must have $x_{\eta_{1}} \geqq x_{\xi}$ for some $\eta_{1}<\eta$. If $\eta_{1}$ is also a limit ordinal, repetition of this argument gives a descending chain of ordinals which terminates with some ordinal $\eta_{k}$, not a limit ordinal, such that $x_{\eta_{k}} \geqq x_{\xi}$. But this is just the preceding case, and thus yields a contradiction. Hence $x_{\eta}<x_{\xi}$ whenever $\eta<\xi<\omega_{\alpha}$. A dual argument on the $y_{\xi}$ 's gives the rest of (5.3).

Now define for each $\xi<\omega_{\alpha}$,

$$
g_{\xi}=b \cup\left(x_{\xi+1} \cap\left(y_{\xi+1} \cup c\right)\right) .
$$

We shall show that the set $\delta=\left\{g_{\xi} \mid \xi<\omega_{\alpha}\right\} \alpha$-generates a sublattice isomorphic to $F_{\alpha}\left(\boldsymbol{\aleph}_{\alpha}\right)$, by verifying the conditions of Theorem 4 for $\mathcal{S}$.

(5.4) For all $\xi<\omega_{\alpha}, g_{\xi} \geqq b, b u t g_{\xi} \geqq a, c$ and $a, b, c \geqq g_{\xi}$.

(5.5) $g_{\xi} \geqq g_{\eta}$ implies $\xi=\eta$.

Statement (5.4) follows easily from the definition of $g_{\xi}$. To show (5.5), suppose that $g_{\xi} \geqq g_{\eta} \geqq x_{\eta+1} \cap\left(y_{\eta+1} \cup c\right) . g_{\xi} \geqq a, c$, and $b \geqq a, c$ imply $x_{\xi+1} \cap\left(y_{\xi+1} \cup c\right)$ $\geqq x_{\eta+1} \cap\left(y_{\eta+1} \cup c\right)$, and this implies that $x_{\xi+1} \geqq x_{\eta+1} \cap\left(y_{\eta+1} \cup c\right)$ and $y_{\xi+1} \cup c$ $\geqq x_{\eta+1} \cap\left(y_{\eta+1} \cup c\right)$. Now since $x_{\xi+1} \geq c$ and $a, b \geq x_{\eta+1} \cap\left(y_{\eta+1} \cup c\right)$, it must be that $x_{\xi+1} \geqq x_{\eta+1}$. Hence $\xi \geqq \eta$. Moreover, since $a, c \geq x_{\eta+1} \cap\left(y_{\eta+1} \cup c\right)$ and $y_{\xi+1} \cup c \geqq a$, it follows that $y_{\xi+1} \cup c \geqq y_{\eta+1} \cup c$, and (5.2) gives $y_{\xi+1} \geqq y_{\eta+1}$. Thus $\xi \leqq \eta$, whence $\xi=\eta$.

(5.6) If $Q \subseteq S$ is such that $|\propto|<\boldsymbol{\aleph}_{\alpha}$, then $\cup a \geqq g_{\xi}$ implies $g_{\xi} \in Q$.

Suppose that $\cup Q \geqq g_{\xi} \geqq x_{\xi+1} \cap\left(y_{\xi+1} \cup c\right)$. If for some $g_{\eta} \in Q, g_{\eta} \geqq x_{\xi+1}$ $\cap\left(y_{\xi+1} \cup c\right)$, then $g_{\eta} \geqq g_{\xi}$, whence $g_{\eta} \equiv g_{\xi}$. This is the only possibility, since $g_{\eta} \geq a, c$ implies $\cup a \geq x_{\xi+1}, y_{\xi+1} \cup c$.

(5.7) If $Q \subseteq S$ is such that $|a|<\boldsymbol{N}_{\alpha}$, then $g_{\xi} \geqq \cap a$ implies $g_{\xi} \in Q$.

If $g_{\xi} \notin a$, then $y_{\xi+1} \cup c \geqq x_{\xi+1} \cap\left(y_{\xi+1} \cup c\right) \geqq \cap a \geqq b$, since $b \geqq \cap a$. But this contradicts (5.1), and hence (5.7) is true.

Notice that up to this point in the proof of Theorem 5, the assumption that $\boldsymbol{\aleph}_{\alpha}$ is regular has not been used. With this assumption, Theorem 4 applies, and Theorem 5 follows from (5.5), (5.6), and (5.7).

THEOREM 6. Let $\alpha$ be any ordinal. If $P$ is a partially ordered set of cardinality at most $\boldsymbol{\aleph}_{\alpha}$, then $F_{\alpha}(P)$ is isomorphic to an $\alpha$-sublattice of $F_{\alpha}(3)$.

Proof. Having Theorem 5, it suffices to embed $F_{\alpha}(P)$ in $F_{\alpha}\left(\boldsymbol{\aleph}_{\alpha}\right)$. Again $\boldsymbol{\aleph}_{\alpha}$ may be assumed regular. 
Let $|P|=\boldsymbol{\aleph}_{\alpha}$, and let $\left\{g_{\xi} \mid \xi<\omega_{\alpha}\right\}$ be the set of $\alpha$-generators of $F_{\alpha}\left(\boldsymbol{\aleph}_{\alpha}\right)$. Then there exists a one-one correspondence

$$
p_{\xi} \leftrightarrow g_{\xi}
$$

between the elements of $P$ and the $\alpha$-generators $g_{\xi}$. Set $a_{0} \equiv g_{0}$. Continuing inductively, let $a_{\eta}$ be defined for each $\eta, \eta<\xi<\omega_{\alpha}$. Then with

define

$$
\begin{aligned}
& \mathcal{U}_{\xi}=\left\{a_{\eta} \mid \eta<\xi, p_{\eta} \geqq p_{\xi} \text { in } P\right\}, \\
& \mathcal{V}_{\xi}=\left\{a_{\eta} \mid \eta<\xi, p_{\xi} \geqq p_{\eta} \text { in } P\right\},
\end{aligned}
$$

$$
a_{\xi} \equiv \cup\left\{\cap\left\{g_{\xi}, u_{\xi}\right\}, v_{\xi}\right\} \text {. }
$$

Since $\left|\mathcal{u}_{\xi}\right|,\left|v_{\xi}\right|<\boldsymbol{\aleph}_{\alpha}, a_{\xi} \in F_{\alpha}\left(\boldsymbol{\aleph}_{\alpha}\right)$ for all $\xi<\omega_{\alpha}$. Let $\jmath=\left\{a_{\xi} \mid \xi<\omega_{\alpha}\right\}$.

(6.1) $a_{\xi} \geqq g_{\eta}$ implies $p_{\xi} \geqq p_{\eta} . g_{\xi} \geqq a_{\eta}$ implies $p_{\xi} \geqq p_{\eta}$.

For $\xi=\eta=0$, (6.1) clearly holds. Inducting on the pairs $\langle\xi, \eta\rangle$, assume that (6.1) holds for any pair less than $\langle\xi, \eta\rangle$. Then if $a_{\xi} \geqq g_{\eta}$, either $g_{\xi} \geqq \bigcap\left\{g_{\xi}, \mathcal{u}_{\xi}\right\}$ $\geqq g_{\eta}$ or $a_{\mu} \geqq g_{\eta}$ for some $a_{\mu} \in \mathcal{V}_{\xi}$. In the first case, $\xi=\eta$ so that $p_{\xi}=p_{\eta}$. In the second, the induction hypothesis implies that $p_{\mu} \geqq p_{\eta}$; whence $p_{\xi} \geqq p_{\mu} \geqq p_{\eta}$. Now suppose that $g_{\xi} \geqq a_{\eta}$. Then $g_{\xi} \geqq \bigcap\left\{g_{\eta}, \mathcal{u}_{\eta}\right\}$ so that either $g_{\xi} \geqq g_{\eta}$ or $g_{\xi} \geqq a_{\mu}$ for some $a_{\mu} \in u_{\eta}$. Thus either $p_{\xi}=p_{\eta}$ or $p_{\xi} \geqq p_{\mu} \geqq p_{\eta}$. And (6.1) follows by induction.

(6.2) $p_{\xi} \geqq p_{\eta}$ implies $a_{\xi} \geqq a_{\eta}$.

When $\langle\xi, \eta\rangle=\langle 0,0\rangle,(6.2)$ is true. If $\xi>\eta$, then $a_{\eta} \in \mathcal{V}_{\xi}$ and hence $a_{\xi} \geqq a_{\eta}$. If $\xi<\eta, a_{\xi} \in \mathcal{u}_{\eta}$, so that $a_{\xi} \geqq \bigcap\left\{g_{\eta}, \mathfrak{u}_{\eta}\right\}$. Thus if (6.2) holds for any pair less than $\langle\xi, \eta\rangle, p_{\xi} \geqq p_{\eta} \geqq p_{\mu}$ implies $a_{\xi} \geqq a_{\mu}$ for all $a_{\mu} \in \mathcal{V}_{\eta}$, and thus $a_{\xi} \geqq a_{\eta}$. Hence (6.2) is true by induction.

(6.3) $a_{\xi} \geqq a_{\eta}$ implies $p_{\xi} \geqq p_{\eta}$.

Let us suppose that (6.3) holds for any pair less than $\langle\xi, n\rangle$. Then if $a_{\xi} \geqq a_{\eta}$, it follows that $a_{\xi} \geqq \bigcap\left\{g_{\eta}, u_{\eta}\right\}$ and four possibilities arise:

(i) $a_{\xi} \geqq g_{\eta}$,

(ii) $a_{\xi} \geqq a_{\mu}$ for some $a_{\mu} \in \mathfrak{u}_{\eta}$,

(iii) $g_{\xi} \geqq \bigcap\left\{g_{\xi}, u_{\xi}\right\} \geqq \bigcap\left\{g_{\eta}, u_{\eta}\right\}$,

(iv) $a_{\xi_{1}} \geqq \bigcap\left\{g_{\eta}, u_{\eta}\right\}$ for some $a_{\xi_{1}} \in v_{\xi}$.

If (i) holds, then $p_{\xi} \geqq p_{\eta}$ follows from (6.1). (ii) and the induction hypothesis imply that $p_{\xi} \geqq p_{\mu} \geqq p_{\eta}$. If (iii) holds, either $g_{\xi} \geqq g_{\eta}$ or $g_{\xi} \geqq a_{\mu}$ for some $a_{\mu} \in \mathfrak{u}_{\eta}$. But then either $p_{\xi}=p_{\eta}$ or $p_{\xi} \geqq p_{\mu} \geqq p_{\eta}$ by (6.1). If (iv) holds, we repeat this procedure obtaining a descending chain of ordinals $\xi=\xi_{0}>\xi_{1}>\cdots>\xi_{i}$ $>\cdots$, such that $a_{\xi_{i+1}} \in V_{\xi_{i}}$. This chain must terminate with some $\xi_{k}$, and at this point (i), (ii), or (iii), but not (iv), holds for $a_{\xi_{k}}$. Thus $p_{\xi_{k}} \geqq p_{\eta}$, and hence $p_{\xi} \geqq p_{\xi_{1}} \geqq \cdots \geqq p_{\xi_{k}} \geqq p_{\eta}$. (6.3) is trivial when $\xi=\eta=0$, so that it must hold for all $\xi, \eta<\omega_{\alpha}$ by induction.

(6.4) If $a \subseteq J$ and $|a|<\boldsymbol{\aleph}_{\alpha}$, then $\cup Q \geqq a_{\xi}$ implies $a_{\eta} \geqq a_{\xi}$ for some $a_{\eta} \in Q$.

Since $a_{0} \equiv g_{0}$, (6.4) holds when $\xi=0$. Inducting on $\xi$, let $a \subseteq J$ be such that $|a|<\mathcal{N}_{\alpha}$ and $\cup a \geqq a_{\xi}$. Then $\cup a \geqq \cap\left\{g_{\xi}, u_{\xi}\right\}$. If $\cup a \geqq g_{\xi}$, then $a_{\eta} \geqq g_{\xi}$ for some 
$a_{\eta} \in Q$, and hence $a_{\eta} \geqq a_{\xi}$ by (6.1) and (6.2). If $\cup Q \geqq a_{\mu}$ for some $a_{\mu} \in \mathfrak{u}_{\xi}$, then by the induction, $a_{\eta} \geqq a_{\mu}$ for some $a_{\eta} \in Q$. But from (6.2), $a_{\mu} \geqq a_{\xi}$, and hence $a_{\eta} \geqq a_{\xi}$. The only remaining possibility is that $a_{\eta} \geqq \bigcap\left\{g_{\xi}, u_{\xi}\right\}$ for some $a_{\eta} \in Q$. In this case, as shown in the proof of (6.3), it follows that $p_{\eta} \geqq p_{\xi}$, and hence that $a_{\eta} \geqq a_{\xi}$. Thus, by induction, (6.4) holds for all $\xi<\omega_{\alpha}$.

(6.5) If $a \subseteq J$ and $|a|<\boldsymbol{\aleph}_{\alpha}$, then $a_{\xi} \geqq \cap a$ implies $a_{\xi} \geqq a_{\eta}$ for some $a_{\eta} \in Q$.

Again (6.5) is clear when $\xi=0$. Continuing with an induction on $\xi$, let $a_{\xi} \geqq \bigcap Q$. If $a_{\mu} \geqq \cap Q, a_{\mu} \in \mathcal{V}_{\xi}$, then by the induction, $a_{\mu} \geqq a_{\eta}$ for some $a_{\eta} \in Q$, and since $a_{\mu} \in V_{\xi}, a_{\xi} \geqq a_{\mu} \geqq a_{\eta}$ by (6.2). If $\cap\left\{g_{\xi}, u_{\xi}\right\} \geqq \cap Q$, then $g_{\xi} \geqq \cap Q$, and hence $g_{\xi} \geqq a_{\eta}$ for some $a_{\eta} \in Q$. This implies that $a_{\xi} \geqq a_{\eta}$, by (6.1) and (6.2). Hence (6.5) holds for all $\xi<\omega_{\alpha}$.

Theorem 6 now follows from (6.2), (6.3), (6.4), (6.5) and Theorem 4.

4. Applications. In this section, some of the results obtained above are used to establish three somewhat unrelated theorems.

TheOREM 7. Every lattice of cardinality at most $\aleph_{\alpha}$ is a sublattice of a lattice $\alpha$-generated by three elements.

Proof. The plan of the proof is to combine the given lattice $L$ with a subset of $F_{\alpha}(3)$ containing its $\alpha$-generators $a, b$, and $c$ and the $\alpha$-generators $g_{\xi}$ of the $\alpha$-sublattice $F_{\alpha}\left(\boldsymbol{N}_{\alpha}\right)$, in such a way that in the resulting partially orered set $\mathfrak{H}, L$ is contained as a sublattice, each element of $L$ is the join of a pair of the $g_{\xi}$, and each element of $\mathfrak{H C}$ can be expressed as an $\alpha$-word over $\{a, b, c\} . \mathcal{F}$ is then completed by cuts to a complete lattice $M$, and this embeds $L$ in the sublattice of $M \alpha$-generated by $\{a, b, c\}$.

Essential to this proof is the idea of the set $s(A)$ of components of an $\alpha$ word $A \in F_{\alpha}(3)$. This set is defined inductively for each $A \in F_{\alpha}(3)$ as follows. For $A \in\{a, b, c\}, s(A)=\{A\}$; if $A \equiv \cup \propto$ or $A \equiv \cap Q$, then

$$
s(A)=\{A\}+\sum_{A^{\prime} \in \mathbb{Q}} s\left(A^{\prime}\right) .
$$

Let $\left\{g_{\xi} \mid \xi<\omega_{\alpha}\right\}$ be the subset of $F_{\alpha}(3)$ constructed in the proof of Theorem 5 which $\alpha$-generates a sublattice isomorphic with $F_{\alpha}\left(\boldsymbol{\aleph}_{\alpha}\right)$, and let

$$
\mathcal{S}=\sum_{\xi<\omega_{\alpha}} s\left(g_{\xi}\right)
$$

(7.1) If $A, g_{\xi} \in \mathcal{G}$ and $A \geqq g_{\xi}$, then $A \equiv g_{\xi}$.

If $a, b, c \geqq A$, then $A \geq g_{\xi}$, by (5.4). If $A \equiv x_{\eta}$, then $A \geq g_{\xi}$ since $x_{\eta} \geq b$. Now suppose that $A \equiv a \cup A^{\prime}$. Then $A^{\prime} \equiv b \cap\left(c \cup A^{\prime \prime}\right)$, where $a \geqq A^{\prime \prime}$, so that $A \geqq g_{\xi}$ implies $c \cup a \geqq A \geqq g_{\xi} \geqq b$, which is impossible. Similarly, if $A \equiv c \cup A^{\prime}$, then $a \geqq A^{\prime}$, and $c \cup a \geq b$ implies $A \geq g_{\xi}$. If $A \equiv b \cup A^{\prime}$, then $A^{\prime}$ has the form $c \cap A^{\prime \prime}$ where $a \cup b \geqq A^{\prime \prime}$. Thus $A \geqq g_{\xi}$, for otherwise, since $A \geqq a$, and $b, c \geqq x_{\xi+1}$ $\cap\left(y_{\xi+1} \cup c\right), A \geqq g_{\xi}$ implies $a \cup b \geqq A \geqq y_{\xi+1} \cup c \geqq c$, an impossibility. Finally, if $A \equiv x_{\eta+1} \cap\left(y_{\eta+1} \cup c\right)$, then $A \geqq g_{\xi}$ implies $a \cup c \geqq y_{\eta+1} \cup c \geqq A \geqq g_{\xi} \geqq b$, which cannot occur. (7.1) now follows, since $g_{\eta} \geqq g_{\xi}$ implies $\eta=\xi$. 
Now suppose that $L$ is a lattice of cardinality $\boldsymbol{\aleph}_{\alpha}$. Then there is one-one correspondence

$$
X_{\xi} \leftrightarrow\left\langle g_{2 \cdot \xi}, g_{2 \cdot \xi+1}\right\rangle
$$

between the elements $X_{\xi}$ of $L$ and the pairs $\left\langle g_{2 \cdot \xi}, g_{2} \cdot \xi+1, \xi \xi<\omega_{\alpha}\right.$. Let $\mathcal{H C}$ be the set $\mathcal{G}+L$, and define a binary relation ( $\geqq)$ over $\mathcal{H}$ as follows.

If $X, Y \in \mathfrak{F}$, then $X \geqq Y$ if one of the following holds:

(i) $X, Y \in L$ and $X \geqq Y$ in $L$,

(ii) $X, Y \in \mathcal{G}$ and $X \geqq Y$ in $F_{\alpha}(3)$,

(iii) $X \in L, Y \in \mathcal{G}$, and there exists a finite subset $\left\{X_{\xi_{0}}, X_{\xi_{1}}, \cdots, X_{\xi_{k}}\right\} \subseteq L$ such that $X \geqq X_{\xi_{0}} \cap \cdots \cap X_{\xi_{k}}$ in $L$ and such that for each $i$, $i \leqq k$, either $g_{2 \cdot \xi_{i}} \geqq Y$ or $g_{2 \cdot \xi_{i}+1} \geqq Y$ in $F_{\alpha}(3)$.

(7.2) The relation ( $\geqq)$ partially orders the set $\mathfrak{H C}$. Moreover, finite meets and joins in $L$ are preserved in $\mathcal{H}$.

(7.3) For each $X_{\xi} \in L, X_{\xi}$ is the least upper bound of $g_{2 \cdot \xi}$ and $g_{2 \cdot \xi+1}$ in $\mathfrak{F}$.

Statement (7.2) is an immediate consequence of the definition of the relation ( $\geqq$ ) over $\mathcal{F C}$. As for (7.3), certainly $X_{\xi} \geqq g_{2 \cdot \xi}, g_{2 \cdot \xi+1}$ in $\mathcal{F C}$. Suppose $Y \in \mathcal{F}$ is such that $Y \geqq g_{2 \cdot \xi}, g_{2 \cdot \xi+1}$ in $\mathfrak{H}$. Then $Y \in L$ and $Y \geqq g_{2 \cdot \xi}$ in $\mathcal{H}$ implies that there exists $X_{\xi_{0}}, \cdots, X_{\xi_{k}} \in L$ such that $Y \geqq X_{\xi_{0}} \cap \cdots \cap X_{\xi_{k}}$, and for each $i \leqq k$, either $g_{2} \cdot \xi_{i} \geqq g_{2} \cdot \xi$ or $g_{2 \cdot \xi_{i}+1} \geqq g_{2} \cdot \xi$. But $g_{\eta} \geqq g_{2 \cdot \xi}$ implies $\eta=2 \cdot \xi$, so that $X_{\xi}=X_{\xi_{0}}=\cdots=X_{\xi_{k}}$, and $Y \geqq X_{\xi}$. Thus $X_{\xi}$ is the least upper bound of $g_{2 \cdot \xi}$ and $g_{2 \cdot \xi+1}$ in $\mathfrak{H C}$.

(7.4) Let $a \subseteq \mathcal{G}$. If $\cap a \in \mathcal{G}$, then $\cap \mathbb{Q}$ is the greatest lower bound of $Q$ in $\mathfrak{F}$; if $\mathrm{U} Q \in \mathcal{G}$, then $\cup a$ is the least upper bound of $a$ in $\mathfrak{F}$.

Suppose that $Q \subseteq \mathcal{G}$ is such that $\cap Q$ is an $\alpha$-word belonging to $\mathcal{G}$. If $B \in \mathcal{H}$ is such that $B \leqq A$ all $A \in Q$, then $B \in \mathcal{G}$ and $B \leqq \cap Q$ in $F_{\alpha}(3)$, so that $\cap Q$ is the greatest lower bound of $Q$ in $\mathcal{F}$. If $a \subseteq \mathcal{G}$ and $U Q$ is an $\alpha$-word in $\mathcal{G}$, and if $B \in \mathcal{G}$ and $B \geqq A$ for all $A \in Q$, then $B \geqq \cup Q$ in $\mathcal{H}$. Suppose that $X \in L$ and $X \geqq A$ for each $A \in Q$. Since $\cup a$ is an $\alpha$-word over $\{a, b, c\}$ belonging to $G$, either $Q=\left\{x_{\eta} \mid \eta<\lambda\right\}$ for some limit ordinal $\lambda<\omega_{\alpha}$, or $Q$ is a two element set. From (5.4), $g_{\xi} \geqq a, c$ for all $\xi$, so that for no $\eta$ can $X \geqq x_{\eta}$ in $\mathcal{H C}$ hold. Thus $Q$ is a two element set $\left\{A_{0}, A_{1}\right\}$ with $A_{0}, A_{1} \geq a, c$. But then for one of these elements, say $A_{0}$, we must have $A_{0} \equiv b$. Thus if $g_{\xi} \geqq A_{1}$, then $g_{\xi} \geqq A_{0} \cup A_{1}$, and hence $X \geqq \cup$.

The partially ordered set $\mathfrak{H}$ is now embedded in a complete lattice $M$ with the MacNeille completion by cuts [5]. Let $N$ be the sublattice of $M$ $\alpha$-generated by $\{a, b, c\}$. Since bounds existing in $\mathcal{H}$ are preserved in $M$, $\mathcal{H C}$ is contained in $N$ by (7.4) and (7.3), and hence by (7.2), $L$ is a sublattice of $N$.

In a recent paper [4], B. Jónsson has considered the existence of an $\boldsymbol{\aleph}_{\alpha^{-}}$ universal lattice, that is, a lattice $L$ of cardinality $\boldsymbol{\aleph}_{\alpha}$, such that every lattice of cardinality at most $\boldsymbol{\aleph}_{\alpha}$ is isomorphic to a sublattice of $L$. He has shown that for all $\alpha>0, \boldsymbol{\aleph}_{\alpha}$-universal lattices do exist. Our next theorem takes up the case $\alpha=0$. 


\section{THEOREM 8. There exist no $\boldsymbol{\aleph}_{0}$-universal lattices.}

Proof. Since any countable lattice can contain only a countable number of finitely generated sublattices, Theorem 8 will follow if we show that there are an uncountable number of nonisomorphic lattices generated by three elements.

Let $\subseteq$ be the set of all sequences $\sigma=\left\langle s_{0}, s_{1}, \cdots, s_{i}, \cdots\right\rangle$ with $s_{i} \in\{0,1\}$, and let $I=\left\{u_{i, j} \mid i<\omega_{0}, j<2\right\}$ be an infinite doubly indexed set. For each $\sigma \in \subseteq$, a partially ordered set $I_{\sigma}$ is obtained by defining a partial ordering over $I$ as follows. $u_{0,0}<u_{1,0}<\cdots<u_{i, 0}<\cdots u_{0,0}<\cdots<u_{i, 0}$ $<u_{i, 1}$ if $s_{i}=0$, or $u_{i, 1}<u_{i, 0}<u_{i+1,0}<\cdots$, if $s_{i}=1$. Clearly, if $\sigma, \tau \in \Im, I_{\sigma}$ and $I_{\tau}$ are isomorphic if and only if $\sigma=\tau$.

As in the proof of Theorem 7, let $\left\{g_{i} \mid i<\omega_{0}\right\}$ generate $F_{0}\left(\boldsymbol{\aleph}_{0}\right)$ in $F_{0}(3)$; let $\mathcal{G}=\sum_{i<\omega_{0}} s\left(g_{i}\right)$; and let $\mathcal{G}^{\prime}=\mathcal{G}+\{a \cap(b \cup c), a \cup(b \cap c), b \cap(a \cup c), b \cup(a \cap c)$, $c \cap(a \cup c), c \cup(a \cap c)\}$. For each $\sigma \in \subseteq$, we construct a partially ordered set $\mathfrak{F}_{\sigma}$ by defining a partial ordering over the set $I+\mathcal{G}^{\prime}$ in the following way.

For $X, Y \in I+\mathcal{G}^{\prime}, X \geqq Y$ if one of the conditions below holds:

(i) $X, Y \in I$ and $X \geqq Y$ in $I_{\sigma}$,

(ii) $X, Y \in \mathcal{G}^{\prime}$ and $X \geqq Y$ in $F_{0}(3)$,

(iii) $X \in I, Y \in \mathcal{G}^{\prime}$ and for some $u_{i j} \in I, X \geqq u_{i j}$ in $I_{\sigma}$, and either $g_{4 i+j} \geqq Y$ or $g_{4 i+j+2} \geqq Y$ in $F_{0}(3)$.

It follows just as in the proof of Theorem 7 that $u_{i, j}$ is the least upper bound in $\mathfrak{F}_{\sigma}$ of $g_{4 i+j}$ and $g_{4 i+j+2}$; if $Q \subseteq \mathcal{G}^{\prime}$ and $\cup Q \in \mathcal{G}^{\prime}$, then $U Q$ is the least upper bound of $Q$ in $\mathfrak{H}_{0}$; and if $a \subseteq \mathcal{G}^{\prime}$ and $\cap a \in \mathcal{G}^{\prime}$, then $\cap Q$ is the greatest lower bound of $a$ in $\mathfrak{H}_{\sigma}$. Thus if the lattice $L_{\sigma}$ is the completion by cuts of $\mathcal{H C}_{\sigma}$, and if $M_{\sigma}$ is the sublattice of $L_{\sigma}$ generated by $\{a, b, c\}$, then $\mathfrak{H}_{\sigma} \subseteq M_{\sigma}$, and bounds existing in $\mathcal{H}_{\sigma}$ are preserved in $M_{\sigma}$.

Notice that in $\mathcal{F}_{\sigma}, X>a$ implies $X \geqq a \cup(b \cap c)$, and $a>X$ implies $a \cap(b \cup c)$ $\geqq X$, with analogous implications holding for $b, b \cup(a \cap c)$ and $b \cap(a \cup c)$, and for $c, c \cup(a \cap b)$ and $c \cap(a \cup b)$. Thus in the completion by cuts, and hence in $M_{\sigma}$, these implications also hold. This implies that $a, b$, and $c$ are both meet and join irreducible in $M_{\sigma}$. Furthermore, since $M_{\sigma}$ is generated by $\{a, b, c\}$, $a, b$, and $c$ are the only elements of $M_{\sigma}$ which have this property. Hence if $\phi$ maps $M_{\sigma}$ isomorphically onto $M_{\tau}$, then $\phi$ maps $\{a, b, c\}$ one-one onto itself. If $\phi$ maps $M_{\sigma}$ isomorphically onto $M_{\tau}$, and $\phi$ induces the trivial permutation on $\{a, b, c\}$, then $\sigma=\tau$. For if $\phi(a)=a, \phi(b)=b$, and $\phi(c)=c$, since meets and joins existing in $\mathfrak{H}_{\sigma}$ and $\mathfrak{H}_{\tau}$ are preserved in $M_{\sigma}$ and in $M_{\tau}$ respectively, it follows that $\phi\left(u_{i, j}\right)=u_{i, j}$ for every $u_{i, j} \in I$. But then $I_{\sigma}$ and $I_{\tau}$ are isomorphic, and hence $\sigma=\tau$. Suppose that in $\left\{M_{\sigma} \mid \sigma \in \mathfrak{S}\right\}$ there is an isomorphism class containing more than six distinct lattices. Since there are only five nontrivial permutations of three elements, in this isomorphism class there must exist three distinct lattices $M_{\rho}, M_{\sigma}$, and $M_{\tau}$, with $\phi_{1}$ and $\phi_{2}$ respectively mapping $M_{\sigma}$ and $M_{\tau}$ isomorphically onto $M_{\rho}$, such that $\phi_{1}(a)=\phi_{2}(a), \phi_{1}(b)=\phi_{2}(b)$, and $\phi_{1}(c)=\phi_{2}(c)$. Then if $\phi=\phi_{2}^{-1} \phi_{1}, \phi$ maps $M_{\sigma}$ isomorphically onto $M_{\tau}$ in such a 
way that $\phi(a)=a, \phi(b)=b$, and $\phi(c)=c$. And as shown above, this implies that $\sigma=\tau$, contrary to the assumption that $M_{\sigma}$ and $M_{\tau}$ are distinct. Thus each

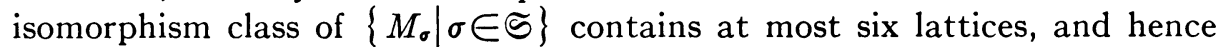
$\left\{M_{\sigma} \mid \sigma \in \subseteq\right\}$ contains $2^{\aleph_{0}}$ nonisomorphic lattices generated by three elements, completing the proof of Theorem 8 .

Associated with the idea of an $\boldsymbol{\aleph}_{\alpha}$-universal lattice is that of an $\boldsymbol{\aleph}_{\alpha}$-universal partially ordered set. $A$ partially ordered set $P$ is said to be $\boldsymbol{\aleph}_{\alpha}$-universal if $|P|=\boldsymbol{\aleph}_{\alpha}$ and if every partially ordered set of cardinality at most $\boldsymbol{\aleph}_{\alpha}$ is isomorphic to a subpartially ordered set of $P$. The lattices $F_{\alpha}(3)$ offer interesting examples of these $\boldsymbol{\aleph}_{\alpha}$-universal partially ordered sets.

THEOREM 9. The free lattice with three generators is an $\boldsymbol{\aleph}_{0 \text {-universal par- }}$ tially ordered set. If the Generalized Continuum Hypothesis holds, then $F_{\alpha}(3)$ is an $\boldsymbol{\aleph}_{\alpha}$-universal partially ordered set if $\boldsymbol{\aleph}_{\alpha}$ is regular, and the sublattice

$$
L_{\alpha}=\left\{A \mid A \in F_{\alpha}(3), r(A)<\omega_{\alpha}\right\}
$$

of $F_{\alpha}(3)$ is an $\boldsymbol{\aleph}_{\alpha}$-universal partially ordered set if $\boldsymbol{\aleph}_{\alpha}$ is singular.

Proof. The sublattice $L_{\alpha}$ will be shown to be an $\boldsymbol{\aleph}_{\alpha}$-universal partially ordered set for all $\alpha$, since when $\boldsymbol{\aleph}_{\alpha}$ is regular, $L_{\alpha}=F_{\alpha}(3)$.

(9.1) If $P$ is a partially ordered set of cardinality at most $\boldsymbol{\aleph}_{\alpha}$, then $P$ is isomorphic to a subpartially ordered set of $L_{\alpha}$.

Let the elements $x_{\xi}, y_{\xi}$, and $g_{\xi}$ of $F_{\alpha}(3)$ be as defined in the proof of Theorem 5. Suppose for all $\eta$ with $\eta<\xi<\omega_{\alpha}, r\left(x_{\eta}\right)=r\left(y_{\eta}\right)=6 \cdot \eta$. If $\xi$ is a limit ordinal, then $r\left(x_{\xi}\right)=r\left(y_{\xi}\right)=\sup _{\eta<\xi}(6 \cdot \eta+1)=\sup _{\eta<\xi} 6 \cdot \eta=6 \cdot \xi$. If $\xi$ is not a limit ordinal, $r\left(x_{\xi}\right)=r\left(y_{\xi}\right)=6 \cdot(\xi-1)+6=6 \cdot \xi$. Thus, since $r\left(x_{0}\right)=r\left(y_{0}\right)=0, r\left(x_{\xi}\right)$ $=r\left(y_{\xi}\right)=6 \cdot \xi$ for all $\xi<\omega_{\alpha}$. Hence if $\xi<\omega_{\alpha}$, then $r\left(g_{\xi}\right)=6 \cdot \xi+9<\omega_{\alpha}$, so that $\left\{g_{\xi} \mid \xi<\omega_{\alpha}\right\} \subseteq L_{\alpha}$. Now let $P$ be a partially ordered set with $|P|=\boldsymbol{\aleph}_{\alpha}$. Let $\left\{a_{\xi} \mid \xi<\omega_{\alpha}\right\}$ be the subset of the $\alpha$-sublattice of $F_{\alpha}(3) \alpha$-generated by $\left\{g_{\xi} \mid \xi<\omega_{\alpha}\right\}$, which was constructed in the proof of Theorem 6 and which is isomorphic to $P$. $a_{0} \equiv g_{0}$, and hence $r\left(a_{0}\right)=9$. Suppose for all $\eta$ with $\eta<\xi<\omega_{\alpha}$, $r\left(a_{\eta}\right) \leqq 6 \cdot \eta+11$. Then for all $\eta<\xi, r\left(a_{\eta}\right)<r\left(g_{\xi}\right)$, whence $r\left(a_{\xi}\right)=r\left(g_{\xi}\right)+2$ $=6 \cdot \xi+11$. Thus $r\left(a_{\xi}\right)<\omega_{\alpha}$, and $\left\{a_{\xi} \mid \xi<\omega_{\alpha}\right\} \subseteq L_{\alpha}$. Hence $P$ is isomorphic to a subpartially ordered set of $L_{\alpha}$.

It is clear that $\left|L_{0}\right|=\boldsymbol{N}_{0}$. To show the corresponding relation for the positive ordinals, the following equivalent form of the Generalized Continuum Hypothesis is needed $\left({ }^{4}\right)$.

If $\alpha$ is any ordinal and if $\mathfrak{M}$ is a set of cardinality $\boldsymbol{\aleph}_{\alpha+1}$, then $\{a \mid a \subset \mathbb{M}$, $\left.|a| \leqq \boldsymbol{N}_{\alpha}\right\}$ has cardinality $\boldsymbol{N}_{\alpha+1}$.

(9.2) If $\alpha$ is not a limit ordinal, then $\left|L_{\alpha}\right|=\boldsymbol{\aleph}_{\alpha}$.

Let $\mathfrak{N}_{0}=\mathfrak{N}_{0}^{\prime}=\{a, b, c\}$, and let $\mathfrak{N}_{\xi}=\left\{A \in L_{\alpha} \mid A \equiv \cup a, r(A)=\xi\right\}, \mathfrak{N}_{\xi}^{\prime}$ $=\left\{A \in L_{\alpha} \mid A \equiv \cap Q, r(A)=\xi\right\}$ for $\xi>0$. To prove (9.2) it suffices to show that

\footnotetext{
${ }^{4}$ See, for example, Bachmann [1, p. 157].
} 
$\left|\Re_{\xi}\right|,\left|\Re_{\xi}^{\prime}\right| \leqq \boldsymbol{\aleph}_{\alpha}$ for each $\xi<\omega_{\alpha}$. If $\left|\Re_{\eta}\right|,\left|\Re_{\eta}^{\prime}\right| \leqq \boldsymbol{\aleph}_{\alpha}$ for all $\eta<\xi$, then $\mathfrak{N}_{\xi}$ $=\sum_{\eta<\xi} \mathfrak{T}_{\eta}+\mathfrak{N}_{\eta}^{\prime}=\left\{A \in L_{\alpha} \mid r(A)<\xi\right\}$ has cardinality at most $\boldsymbol{\aleph}_{\alpha}$. Now if $A \in \mathscr{N}_{\xi}$ with $A \equiv \cup a$, then $a \subseteq \mathscr{T}_{\xi}$ and $|a| \leqq \boldsymbol{N}_{\alpha-1}$. Thus the cardinality of $\mathscr{N}_{\xi}$ cannot exceed the cardinality of $\left\{Q\left|a \subseteq \mathfrak{N}_{\xi},\right| Q \mid \leqq \boldsymbol{N}_{\alpha-1}\right\}$, and hence if the Generalized Continuum Hypothesis holds, $\left|\mathfrak{N}_{\xi}\right| \leqq \boldsymbol{\aleph}_{\alpha}$. Similarly, $\left|\mathfrak{N}_{\xi}^{\prime}\right|$ $\leqq \boldsymbol{\aleph}_{\alpha}$.

(9.3) If $\alpha$ is a limit ordinal, then $L_{\alpha}=\sum_{\beta<\alpha} L_{\beta}$. Hence $\left|L_{\alpha}\right|=\boldsymbol{\aleph}_{\alpha}$.

If $\beta<\alpha$, every $\beta$-word over $\{a, b, c\}$ is also an $\alpha$-word over $\{a, b, c\}$, and hence $L_{\alpha} \supseteq \sum_{\beta<\alpha} L_{\beta}$. We now show that if $A \in L_{\alpha}$ and $r(A) \leqq \omega_{\beta}$ then $A \in L_{\beta}$. If $r(A)=0$, this clearly holds. Inducting on $r(A)$, let $A \equiv \cup \propto$ or $\cap a$. Since $r(A)<\omega_{\beta}, r\left(A^{\prime}\right)<\omega_{\beta}$ for each $A^{\prime} \in Q$, and hence $A^{\prime} \in L_{\beta}$ all $A^{\prime} \in Q$, by the induction hypothesis. Also, $r(A)<\omega_{\beta}$ implies $|Q|<\boldsymbol{\aleph}_{\beta}$. Thus $A$ is a $\beta$-word over $\{a, b, c\}$, and $A \in L_{\beta}$. Now if $A \in L_{\alpha}, r(A)<\omega_{\alpha}$, and since $\alpha$ is a limit ordinal, $\beta$ exists such that $r(A)<\omega_{\beta}<\omega_{\alpha}$, and hence $A \in L_{\beta}$. Thus $L_{\alpha} \subseteq \sum_{\beta<\alpha} L_{\beta}$, and (9.2) follows.

This completes the proof of Theorem 9 .

\section{REFERENCES}

1. H. Bachmann, Transfinite Zahlen, Ergebnisse der Mathematik und ihrer Grenzgebiete (N.F.) vol. 1, Springer-Verlag, Berlin, 1955.

2. R. A. Dean, Completely free lattices generated by partially ordered sets, Trans. Amer. Math. Soc. vol. 83 (1956) pp. 238-249.

3. R. P. Dilworth, Lattices with unique complements, Trans. Amer. Math. Soc. vol. 57 (1945) pp. 123-154.

4. Bjarni Jonsson, Universal relational systems, Math. Scand. vol. 4 (1956) pp. 193-208.

5. H. M. MacNeille, Partially ordered sets, Trans. Amer. Math. Soc. vol. 42 (1937) pp. 416-460.

6. P. M. Whitman, Free lattices I, Ann. of Math. (2) vol. 42 (1941) pp. 325-330.

7. - Free lattices II, Ann. of Math. (2) vol. 43 (1941) pp. 104-115.

California Institute of Technology, Pasadena, California 